Supplementary Information (SI)

\title{
Nanocomposites of Spiropyran-Functionalized Polymers and Upconversion Nanoparticles for Controlled Release Stimulated by Near-Infrared Light and pH
}

Shuo Chen, ${ }^{\text {a,c }}$ Yujuan Gao, ${ }^{\mathrm{b}}$ Ziquan Cao, ${ }^{\mathrm{a}}$ Bo Wu, ${ }^{\mathrm{a}}$ Lei Wang, ${ }^{\mathrm{b}}$ Hao Wang,*b Zhimin Dang, ${ }^{c}$ Guojie Wang*a

${ }^{a}$ School of Materials Science and Engineering, University of Science and Technology Beijing, Beijing 100083, China;

${ }^{b}$ Laboratory of Biological Effects of Nanomaterials and Nanosafety National Center for Nanoscience and Technology (NCNST) Chinese Academy of Sciences, Beijing 100190 china;

${ }^{c}$ Department of Polymer Science and Engineering, University of Science and Technology Beijing, Beijing 100083, China;

AUTHOR EMAIL ADRESS: guojie.wang@mater.ustb.edu.cn; wanglei@nanoctr.cn; wanghao@nanoctr.cn;dangzm@ustb.edu.cn 


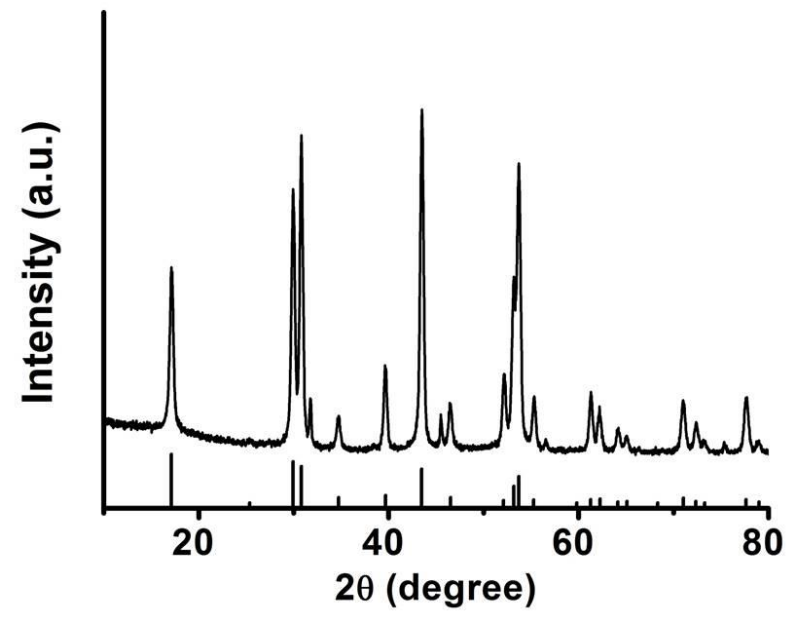

Fig. S1 XRD pattern of the prepared UCNPs and the corresponding standard data of $\mathrm{NaYF}_{4}$ (JCPDS No. 16-0334).

Fig. S1 shows the X-ray diffraction patterns of the prepared UCNPs. All diffraction peaks match well with the standard pattern of hex-agonal-phase $\mathrm{NaYF}_{4}$ (JCPDS No. 16-0334) ${ }^{[48]}$. The hexagonal-phase revealed by XRD is consistent with the morphology observation of TEM image (Figure 1a). 

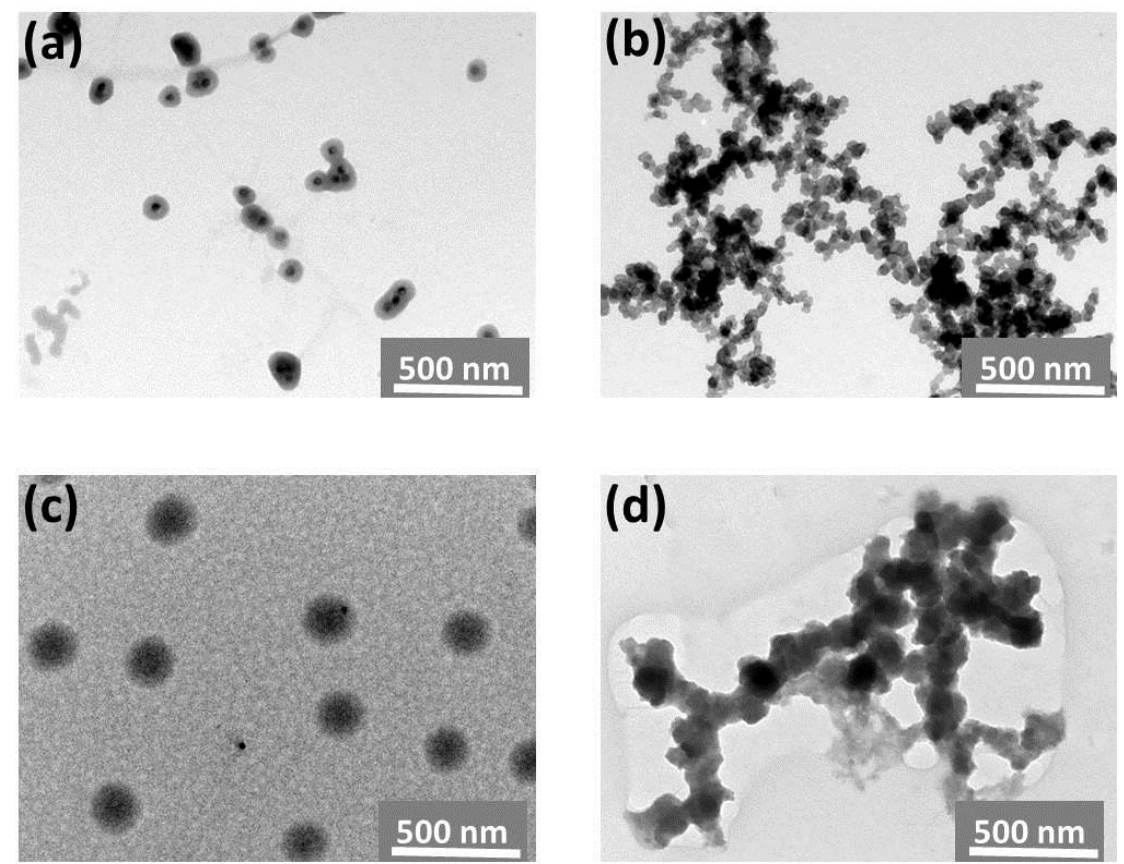

Fig. S2 TEM images of UCNPs @ Polymer: a) without stimulation; b) under NIR irradiation; c) at $\mathrm{pH}=5.0 ; \mathrm{d})$ under NIR and $\mathrm{pH}=5.0$ combined stimulation.

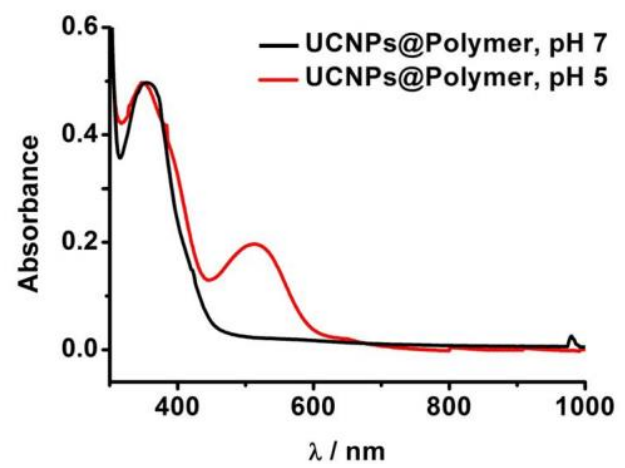

Fig. S3 UV-Vis absorption spectra of UCNPs@ Polymer nanocomposites under different $\mathrm{pH}$ stimulation.

Fig. S3 shows the UV-vis spectra of the UCNPs@Polymer nanocomposites under different $\mathrm{pH}$ stimulation, from which it can be seen that the absorption peak centered 
at $980 \mathrm{~nm}$ corresponding to that of UCNPs disappeared at pH 5 after $24 \mathrm{~h}$. At acidic condition, the hydrophobic SP could isomerize to hydrophilic MC and the polymer self-assemblies were swelled to larger ones and the hydrophobic oleate-capped UCNPs would be released from the nanocomposites.
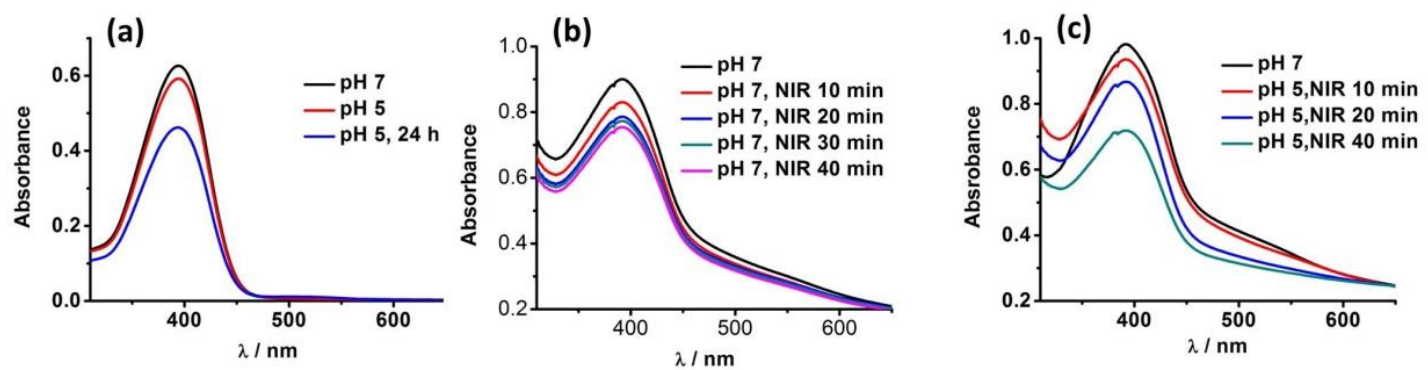

Fig. S4 UV-Vis absorption spectra of Coumaran 102 loaded in UCNPs@ Polymer nanocomposites: a) at $\mathrm{pH}=5.0$; b) under NIR irradiated; c) under NIR and $\mathrm{pH}=5.0$ combined stimulation.
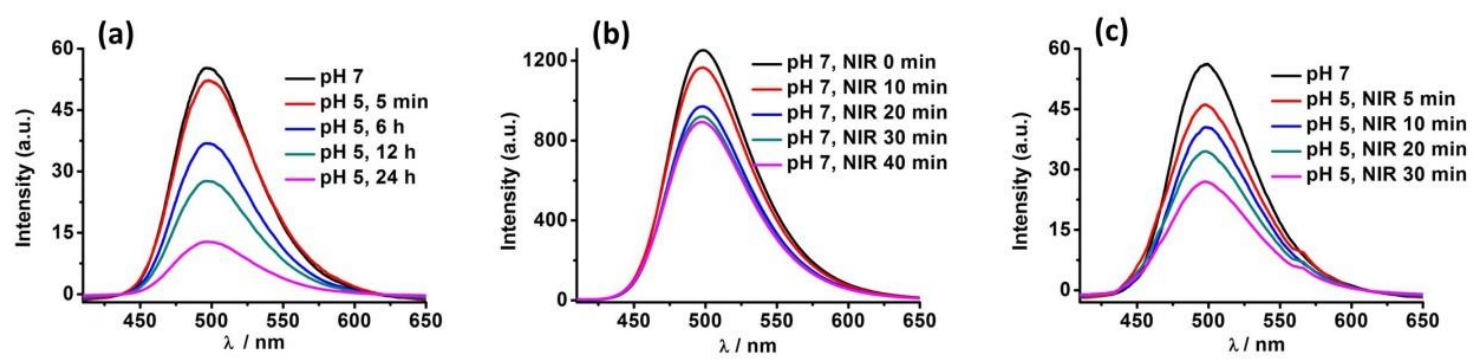

Fig. S5 Emission spectra of Coumaran 102 loaded in UCNPs@Polymer nanocomposites: a) at $\mathrm{pH}=5.0$; b) under NIR irradiation; c) under NIR and $\mathrm{pH}=5.0$ combined stimulation. 

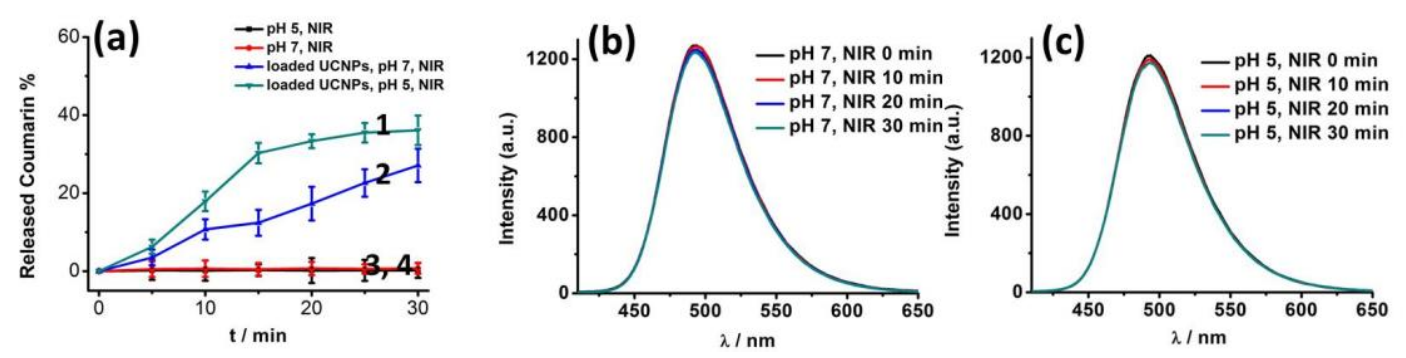

Fig. S6 a) Release profiles of Coumarin 102 loaded in the micellar nanoparticles with UCNPs $(1,2)$ and loaded in the micellar nanoparticles without UCNPs $(3,4)$ under NIR irradiation for $30 \mathrm{~min}$ at different $\mathrm{pH}$ values; b) and c) Emission spectra of Coumaran 102 loaded in micellar nanoparticles without UCNPs under NIR irradiation at $\mathrm{pH} 7$ and $\mathrm{pH} 5$, respectively.

Figure S6 shows that the release of loaded molecules from the polymer nanoparticles without UCNPs is very little although the NIR irradiation could increase the temperature of the system to about $50^{\circ} \mathrm{C}$.

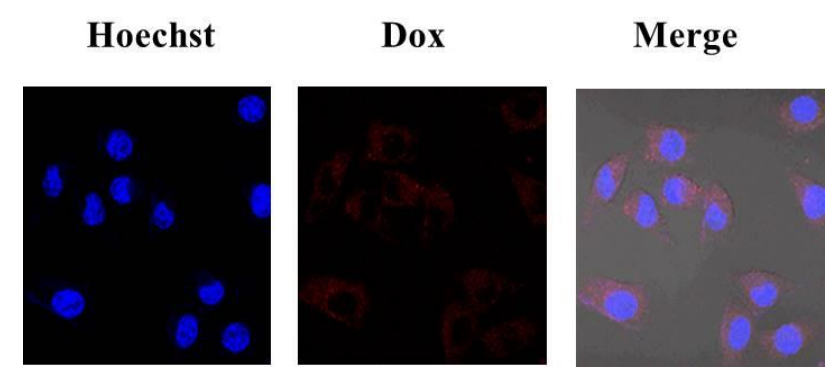

Fig. S7 Confocal laser scanning microscope observations of the Dox release in U-87 MG cells treated with Dox-loaded UCNPs@Polymer nanocomposites incubated for 2 $\mathrm{h}$ without NIR light irradiation. For each panel, the images from left to right show cell nuclei stained by Hoechst (blue; Hoechst 33342), Dox fluorescence in cells (red) and overlays of the two images. 